\title{
Highly deformed grain: from the Hertz contact limitation to a new strain field description in 2D
}

\author{
Serge Mora ${ }^{1, \star}$, Thi-Lo Vu ${ }^{1, \star \star}$, Jonathan Barés ${ }^{1, \star \star \star}$, and Saeid Nezamabadi ${ }^{1, \star \star \star \star}$ \\ ${ }^{1}$ Laboratoire de Mécanique et Génie Civil, Université de Montpellier, CNRS, Montpellier, France
}

\begin{abstract}
.
Most of the 2D studies on granular materials consider an assembly of cylindrical particles in the small deformation regime. Investigating the range of validity of the small deformation hypothesis is therefore of crucial importance in order to determine the quantitative relevance of the predictions emerging from these 2D studies. We introduce here a novel experimental set-up based on high resolution imaging and image correlation, and capable of measuring the strain field inside the particles with a cutting edge accuracy. We measure the strain field inside a unique circular cylindrical elastic particle undergoing an uniaxial compression. The significant deviations arising from the comparison with the predictions of the small deformation limit predicted by the Hertz's theory, and numerical simulations, give insight to improve the modeling of 2D granular materials.
\end{abstract}

\section{Introduction}

When local forces are measured or computed in granular matter physics, whether it is for numerical [1-3] or experimental studies [4-6], collections of circular grains with low polydispersity and in small deformation regime are very commonly considered. This permits to conform with the Hertz contact law [7] which, along with the superposition principle, provides the most common mechanical description of a grain contact. Unfortunately in real life most of the granular problems involve unevenly shaped grains, on a very broad range of sizes, in highly stressed situations [8-11]. The lack of a net demarcation line separating the regime of small deformations with a regime where it is necessary to take into account large deformations within the grains is a source of confusion. For example in the case of jammed granular systems some grains (the one involved in force chains [1]) can be far above the small deformation hypothesis which ruins the macroscopic description of the whole system.

Hence, in order to observe, model and simulate properly realistic granular media it is necessary to get a complete description of a grain behaviour beyond the linear elastic limit. This would permit to check already existing numerical models [12-14] and to provide inputs for improvements.

By means of novel 2D experimental approaches, we investigate when the Hertz strain field [7] is not valid any more and how it has to be modified beyond linear elasticity. To do so we introduce a new experimental measure-

\footnotetext{
$\star$ e-mail: serge.mora@umontpellier.fr

$\star \star$ e-mail: thi-lo.vu@umontpellier.fr

$\star \star \star$ e-mail: jb@jonathan-bares.eu

$\star \star \star \star$ e-mail: saeid.nezamabadi@umontpellier.fr
}

ment method suitable for granular material. We set-up a step by step single disk radial compression experiment attached to a very high resolution imaging apparatus $(5 \mu \mathrm{m}$ accuracy over a $6 \mathrm{~cm}$ diameter disc particle). The disk is one base surface of a thin cylinder made in an hyperelastic material. The displacement field in the plane of the disk is then precisely measured both spatially and in intensity via Digital Image Correlation (DIC). Strain is directly deduced and compared with theoretical results. The limitations arising from the linear elastic theory are then highlighted, for this common mechanical situation. These results are also compared to what is obtained by finite element (FE) simulations.

In the first part of this article, we present the experimental set-up, how we compress a particle and how we image it. Then, in a second part we give some image processing details to measure the displacement field inside the particle. Strain field is computed and compared to what is obtained with FE. Finally, in a last part, we present some results, conclusions and perspectives.

\section{Experimental set-up and material}

A manufactured in-house black silicone cylindrical particle [15] (Young modulus 2.7MPa) coated on one side with shiny very thin glitter [16] (average size $30 \mu \mathrm{m}$ ) is led on the top glass of a flatbed scanner as presented in fig. 1-A. The initial diameter of the particle is $5.9 \mathrm{~cm}$ and its thickness is $9.5 \mathrm{~mm}$. As presented in fig. 1-B the glitter coating forms an homogeneous thin random pattern on the bottom face of the particle with a high black/white contrast. Step by step, the flat cylinder is squeezed in its radial direction in a quasistatic manner by a manufactured in-house uniaxial compression apparatus (see fig. 1-A). This device 


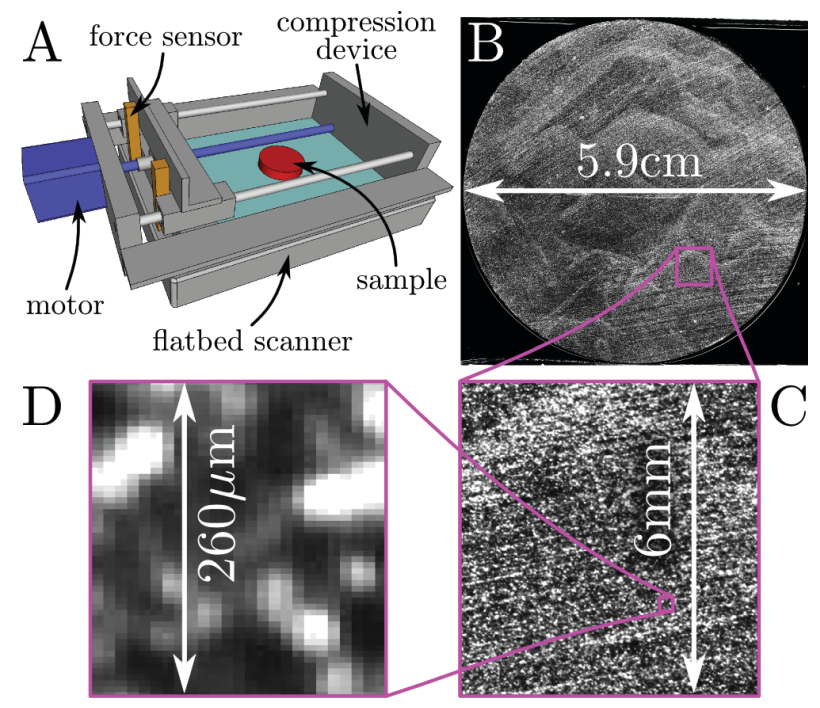

Figure 1. A: Sketch of the experimental set-up. A cylindrical particle ( $5.9 \mathrm{~cm}$ diameter and $9.5 \mathrm{~mm}$ thickness) lays on a flatbed scanner. A manufactured in-house compression device squeezes the particle step by step while the particle is imaged from below and the compression force is measured. B: Black and white scanner bottom view of the particle. A thin pattern has been deposited on the particle. C: Zoomed view of the particle pattern. D: Zoomed view to the size of a correlation length. The pixel size is $5.29 \mu \mathrm{m}$.

is accurately actuated by means of a stepper motor and a threaded rod with a very low distance/step ratio. The force is measured with two $1 \mathrm{~kg}$ force cells. Vegetable oil with a very low viscous coefficient (60 mPa.s) is coated on the scanner glass to almost vanish static basal friction and improve optic transmission.

After each $0.5 \mathrm{~mm}$ compression step, the system is imaged with the flatbed scanner. As emphasized by the results presented in this paper, it constitutes a very stable and accurate measurement apparatus both in space (tunable from 70dpi to 9600dpi) and numerical depth (tunable from 8bits to 16bits for each color) on a large area (maximum $21 \times 29.7 \mathrm{~cm}^{2}$ ) without any optical distortion on a flat area. For the results presented in this paper, we used a scanner CanoScan 9000F Mark $I I$ with resolution $4800 \times 4800 \mathrm{dpi}^{2}$ on a $6 \times 6 \mathrm{~cm}^{2}$ area on 8 bits for black and white picture. As presented in fig. 1-C \& D, we image the particle bottom on a wide area with an extreme accuracy.

\section{Image post-processing}

Image correlation for granular material has been widely used both in 2D and 3D at the continuum scale [17-21] to measure the grain flow but to our knowledge it has never been used at the scale of each grain to measure their deformation. However, as emphasized earlier in this paper it is of high interest to study stress networks in the most common granular media: unevenly shaped, highly stressed or deformed grains with any rheological behaviour. Until now, the only way to measure force between grains was to use photoelasticity [4, 22], thermoelasticity [5] or

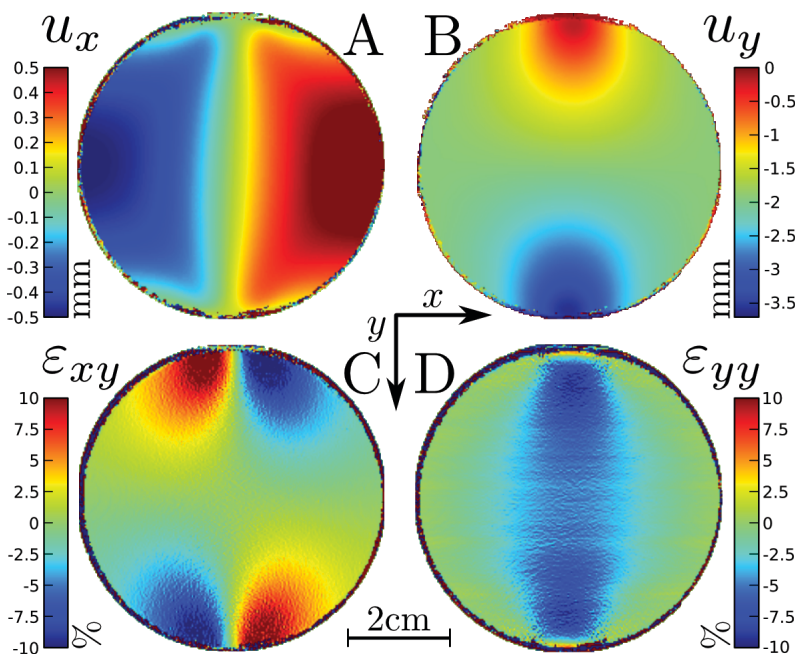

Figure 2. A,B: Displacement field measured from the local image correlation along the $x$ and $y$ direction respectively. C, D: $\varepsilon_{x y}$ and $\varepsilon_{y y}$ components (respectively) of the strain field. Fields are plotted on the undeformed geometry for a $8 \%$ total deformation of the particle. Measurement are noisy at the boundary because the correlation cells overlap patterned and unpatterned domains. The different fields are plotted on the undeformed coordinates (Lagrangian).

boundary deformation [6]. However for each of these techniques, particle materials and shapes are very restricted and it can be performed only in the small deformation regime. Here we propose a method capable of measuring the full stress field for any kind of grain and any loading in $2 \mathrm{D}$.

By means of digital image correlation (DIC) technique $[23,24]$, adapted to high image size and large deformations we measure the evolution of the displacement field inside a compressed silicon particle. A regular mesh of $260 \times 260 \mu \mathrm{m}^{2}\left(50 \times 50 \mathrm{px}^{2}\right)$ is first built on the undeformed initial picture. For each cell of the mesh, also called correlation cell, the displacement along $x$ and $y$ directions is measured incrementally between the picture of each compression step and the undeformed picture. To do so, a first measurement is done, with a 1px accuracy, using a Fourier transformed based algorithm [24]. Then, the measurement is improved to subpixel accuracy by means of computationally heavier algorithm based on an optimization algorithm [25] maximizing the correlation between deformed pictures and the undeformed one. To take the large deformations into account both algorithmic steps are performed sequentially for correlation cells going from 3 times bigger to $50 \times 50 \mathrm{px}^{2}$. Parallelized on $12,3 \mathrm{GHz}$ nodes, the computation take around $\sim 6$ hours for 30 compression steps.

\section{Results and discussions}

As shown in fig. 2-A \& B for a global compression of $8 \%$ strain, the $x$ and $y$ components of the displacement, $u_{x}$ and $u_{y}$ ( $y$ is for the direction of the global compression, $x$ is for the in-plane orthogonal direction) are measured with 

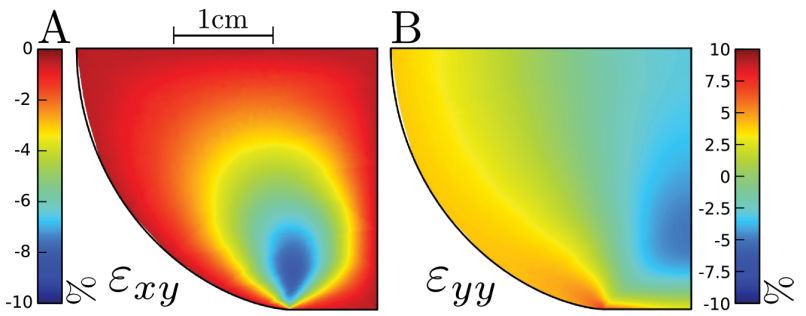

Figure 3. Computed strain. A, B: $\varepsilon_{x y}$ and $\varepsilon_{y y}$ components (respectively) of the strain field computed by FE using Cast3M [26]. Using symmetries only $1 / 4$ of the the particle is simulated. The material is supposed to be linear, elastic and isotropic for a global $8 \%$ compression. Fields are plotted on the deformed system (Eulerian). Results are qualitatively similar to what is found experimentally. Difference is explained because the system is at the limit of the small deformation hypothesis.

an excellent smoothness and accuracy compared to what is classically done in the small deformation domain for disc compression [23]. Nevertheless around the narrow particle boundary, data are noisy since correlation cells overlap both particle and unpatterned surrounding space: correlation information in the particle is mixed with surrounding black area where no correlation is possible. Hence for these correlation cells displacement information is less accurate than displacement for cells in the middle of the particle. Deriving these raw displacement data $u_{x}$ and $u_{y}$, as shown in fig. 2-C \& D we then compute the strain inside the particle, $\varepsilon_{x y}=\frac{\partial u_{x}}{\partial y}+\frac{\partial u_{y}}{\partial x}$ and $\varepsilon_{y y}=\frac{\partial u_{y}}{\partial y}$ respectively. It is important to note that these data are obtained without any smoothing nor filtering at a scale higher than the cell scale. As expected from Hertz's theory [7], we find (from $\varepsilon_{y y}$ ) that the strain parallel to the compression direction is symmetric and maximum in a thin ellipse joining the two contact points. In fig. 2-D we also observed slight horizontal changes in the blue shade. They correspond with small instability of the scanner motor speed. This is the only artefact we have noticed coming from the scanner and it can be put in perspective to the fact that no noise filtering has been applied to the pictures nor to the post-processed data. Similarly, for $\varepsilon_{x y}$, results qualitatively in agreement with Hertz's theory are observed since the shear strain is anti-symmetric with two opposite lobes going from the contact areas. Then, assuming a linear rheology, the stress field is computed from the measured strain field with a Young modulus of 2.7MPa and a Poisson ratio of 0.49 (not shown).

In parallel to this, numerical simulations have been carried out, by means of the FE code Cast3M [26]. Taking advantage of the symmetries, $1 / 4$ of the particle is simulated in the same mechanical condition as the experiments except the material is considered as linear, elastic and isotropic for the full deformation range. As presented in fig. 3-A \& B the results are in qualitative agreement with what is measured experimentally but strain intensity do not seem to be the same.

In fig. 4-A, we show $\varepsilon_{y y}(y)$ the compression strain value along the loading direction. This has been plotted

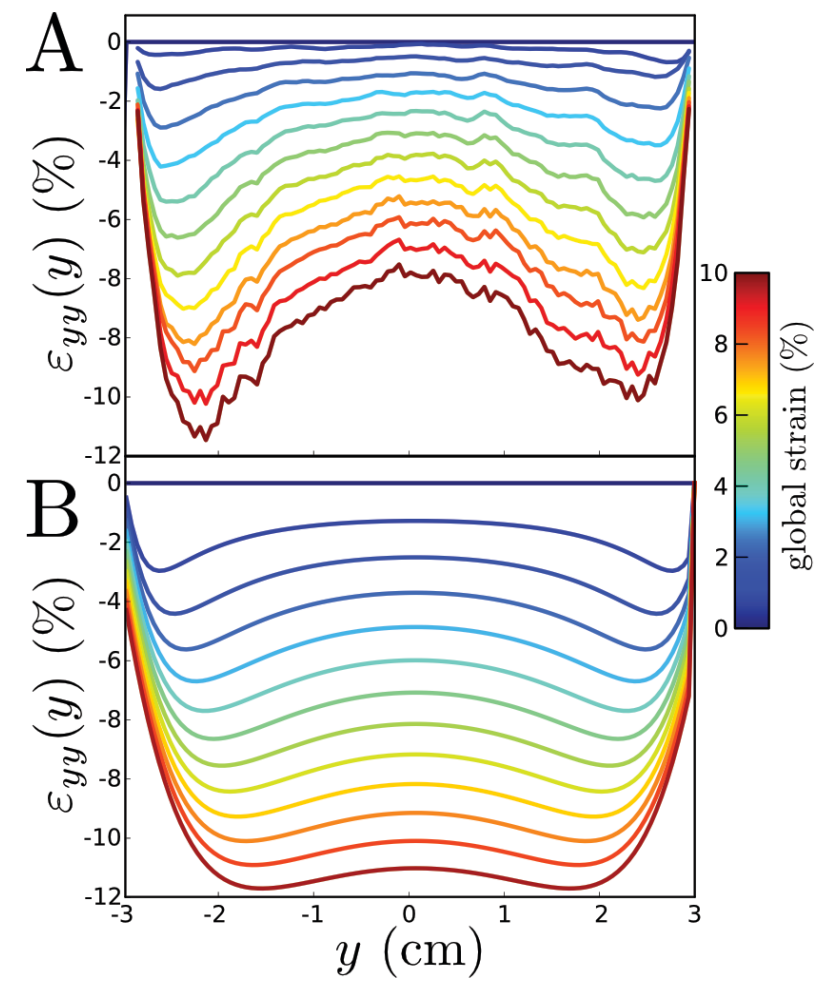

Figure 4. Strain evolution during the compression process along the $y$-direction. A,B: Evolution of the strain component $\varepsilon_{y y}(y)$ as function $y$, along the diameter parallel to $y$, for different compression levels going from $0 \%$ to $10 \%$ in the experimental case (A) and computed from Hertz's contact law (B). $y=0$ corresponds to the center of the specimen. For the experiment, data close to the contact point have been cropped because they are noisy. The curve shape is sensibly different for large deformations.

for different compression steps. We see that this value vanishes at the contact point, rapidly increases toward the center of the particle and then decreases before reaching a plateau in the middle of the sample. As presented in fig. 4-B, this is consistent with what is predicted by Hertz contact law for small deformations. Also, even for large deformations the minimum value of the strain near the contact points is well predicted. However when the global loading is larger than few percent, fig. 4-AB shows that the strain is quantitatively different in the center of the disc: the strain is largely overestimated. This can be explained by the fact that small deformation hypothesis is not valid any more or because out of plan displacement cannot be neglected any more in the experiment.

\section{Conclusion and perspectives}

In conclusion, in this paper we have presented an original experimental apparatus capable of compressing cylindrical granular particles while imaging them with an excellent accuracy. Along with a manufactured in-house fast DIC algorithm adapted to take large deformation into account, the evolution of the displacement field inside the particle is measured with an edge cutting accuracy. Strain field has been deduced and compared to what is expected 
from Hertz's contact law. A fairly good qualitative agreement have been observed as long as the system stays in the small deformation regime.

This paper validates an experimental method for the measurement of the full strain and stress field at the grain scale for any loading and opens a new front of experimental exploration for a wide range of granular material: soft, brittle, mixed, etc. Hence in future works, the experimental measurement will be extended to different model materials: plastically deformable material, brittle material, zero Poisson ratio material,etc. The results will be compared to the ones obtained with different numerical models (finite element method for example) for different boundary conditions. The goal will be to show the limitation of these numerical models either by the fact they do not take the full complex rheology into account or by the fact they are limited in terms of spacial discretisation of the particles (for the bulk or for the contact model). This method can also be extended to $3 \mathrm{D}$ measurements in granular matter using tomography imaging.

\section{Acknowledgements}

This work is supported by the Labex NumEv (anr-10-labx20).

\section{References}

[1] F. Radjai, M. Jean, J.J. Moreau, S. Roux, Physical Review Letters 77, 274 (1996)

[2] F. da Cruz, S. Emam, M. Prochnow, J.N. Roux, F. Chevoir, Physical Review E 72, 021309 (2005)

[3] T. Bertrand, R.P. Behringer, B. Chakraborty, C.S. O’Hern, M.D. Shattuck, Physical Review E 93, 012901 (2016)

[4] T.S. Majmudar, R.P. Behringer, Nature 435, 1079 (2005)

[5] P. Jongchansitto, X. Balandraud, M. Grediac, C. Beitone, I. Preechawuttipong, Soft Matter 10, 8603 (2014)

[6] N. Brodu, J.A. Dijksman, R.P. Behringer, Nature communications 6 (2015)

[7] K.L. Johnson, Contact mechanics (Cambridge university press, 1987)

[8] G. Romeo, M.P. Ciamarra, Soft Matter 9, 5401 (2013)
[9] N. Ouhbi, C. Voivret, G. Perrin, J.N. Roux, Procedia Engineering 143, 1120 (2016), advances in Transportation Geotechnics $\{\mathrm{III}\}$

[10] S.A. Galindo-Torres, D.M. Pedroso, D.J. Williams, L. Li, Computer Physics Communications 183, 266 (2012)

[11] P. Menut, S. Seiffert, J. Sprakel, D.A. Weitz, Soft Matter 8, 156 (2012)

[12] É. Azéma, F. Radjaï, R. Peyroux, G. Saussine, Physical Review E 76, 011301 (2007)

[13] V. Topin, F. Radjai, J.Y. Delenne, F. Mabille, Powder Technology 190, 215 (2009), selection of Papers from the Symposium Powder Science and Technology - Powders and Sintered Material STP-PMF 2007 Symposium on Powder Science and Technology Powders and Sintered Material STP-PMF 2007

[14] S. Nezamabadi, F. Radjai, J. Averseng, J.Y. Delenne, Journal of the Mechanics and Physics of Solids 83, 72 (2015)

[15] Smooth-On ${ }^{\circledR}$, Mold Star ${ }^{\circledR} 15$ SLOW colored with few drop of black Silc Pig ${ }^{\circledR}$

[16] Smooth-On ${ }^{\circledR}$, Cast Magic ${ }^{\circledR}$ (silver bullet)

[17] S.A. Hall, D.M. Wood, E. Ibraim, G. Viggiani, Granular matter 12, 1 (2010)

[18] S.A. Hall, M. Bornert, J. Desrues, Y. Pannier, N. Lenoir, G. Viggiani, P. Bésuelle, Geotechnique 60, 315 (2010)

[19] A.L. Rechenmacher, S. Abedi, O. Chupin, A.D. Orlando, Acta Geotechnica 6, 205 (2011)

[20] E. Ando, S.A. Hall, G. Viggiani, J. Desrues, P. Bésuelle, Acta Geotechnica 7, 1 (2012)

[21] C. Slominski, M. Niedostatkiewicz, J. Tejchman, Powder Technology 173, 1 (2007)

[22] M. Cox, D. Wang, J. Barés, R.P. Behringer, Europhysics Letters (2016)

[23] F. Hild, S. Roux, Strain 42, 69 (2006)

[24] B. Pan, K. Qian, H. Xie, A. Asundi, Measurement science and technology 20, 062001 (2009)

[25] M. Bornert, F. Brémand, P. Doumalin, J.C. Dupré, M. Fazzini, M. Grédiac, F. Hild, S. Mistou, J. Molimard, J.J. Orteu et al., Experimental Mechanics 49, 353 (2009)

[26] Cast3M, CEA, http://www-cast3m.cea.fr 